\title{
Gamma Ray Spectrometer Data Processing Technique to Reduce the Influence of Statistical Fluctuation and Random Noise
}

\author{
Krishna Patel \\ Master of Technology \\ Department of Computer \\ Engineering \\ CSPIT, CHARUSAT
}

\author{
Dipak Kumar Panda \\ Scientist-SE \\ Planetary Science Division \\ Physical Research Laboratory- \\ Ahmedabad (Navrangpura), \\ Gujarat
}

\author{
Ritesh Patel \\ Associate Professor \\ Department Of \\ Computer Engineering \\ CSPIT, CHARUSAT
}

\begin{abstract}
The paper introduces smoothing method for gamma ray spectra. Motive of this method is to reduce the influence of statistical fluctuations and the random noise. Because of fluctuation, data prohibit for accurate estimation of composition of sample. In this work we have used the laboratory based data obtained from the Gamma Ray Spectrometer (GRS) developed in our laboratory using different scintillation detector like $\mathrm{LaBr}_{3}: \mathrm{Ce} / \mathrm{CeBr}_{3}$. The digitized data from the developed GRS instrument are readout to computer through a NI DIO (National Instruments Digital Input/Output) card. Data acquisition system has been developed in LabVIEW to generate the gamma spectrum.
\end{abstract}

\section{Keywords}

Gamma Rays, Smoothing, Noise.

\section{INTRODUCTION}

Gamma ray spectrometer (GRS) is a well-established technique to find out the elemental abundance. The development design of the GRS is mainly divided into four parts: (i) front-end electronics; (ii) biasing module; (iii) data processing unit, and (iv) data acquisition system. GRS can provide the elemental abundance of elements major elements like $\mathrm{Ti}, \mathrm{Al}, \mathrm{O}, \mathrm{Fe}, \mathrm{Si}, \mathrm{Th}, \mathrm{U}, \mathrm{Mg}, \mathrm{K}$ and $\mathrm{Ca}$ which are the main components for the formation of rock, sediment and soil. A gamma-beam spectrometer is an instrument that measures the scattering of the force of gamma radiation versus the essentialness of every photon. ${ }^{[2]}$ The elemental abundance can be used to deduce the chemical composition. The surface elemental composition can be used to understand the origin and evolution of planetary bodies. High energy gamma-ray spectroscopy can be used for remote sensing to find out the chemical composition of planetary surface and has been used to study surface composition of the Moon, Mars and asteroids at spatial resolution.

Various missions carrying gamma ray spectrometer has been flown to moon, mars, mercury and asteroid bodies. Lunar Prospector has been flow to moon and has accumulated information over the whole surface of the moon. Similarly, in 2001 Mars Odyssey has been flown on Mars. Lunar Prospector has been flown with a Bismuth Germanate (BGO) with a plastic scintillator as an anti-coincidence detector while Mars Odyssey has carried a High Purity Germanium (HPGe). In our laboratory GRS has been developed using different scintillation crystals like $\mathrm{LaBr}_{3}: \mathrm{Ce}$ and $\mathrm{CeBr}_{3}$. Data acquisition system has been developed in LabVIEW to interpret the data from the instrument.
The gamma ray spectra obtained from the GRS instruments is not a smooth one; it includes random noise and fluctuation. To have good informative data these spectra has to be smoothen using some method. In this work our objective is to extricate the valuable data from the deliberate information while limiting the impact of measurable variance that can raise figuring mistake on both nuclide acknowledgment and quantitative investigation $^{[1]}$.

\section{PROPOSED APPROACH}

To generate a gamma ray spectrum from gamma ray spectrometer a sequence of step are involved like detection of gamma event by the detector, conversion of these events into digital format by processing electronics and finally these digital data are read out by computer through a data acquisition card. The gamma ray spectrum generated is in the form of count vs. channel, the number of channels available in spectrum is 8192. In generated spectra there is a many fluctuations available which is shown in figure (a).

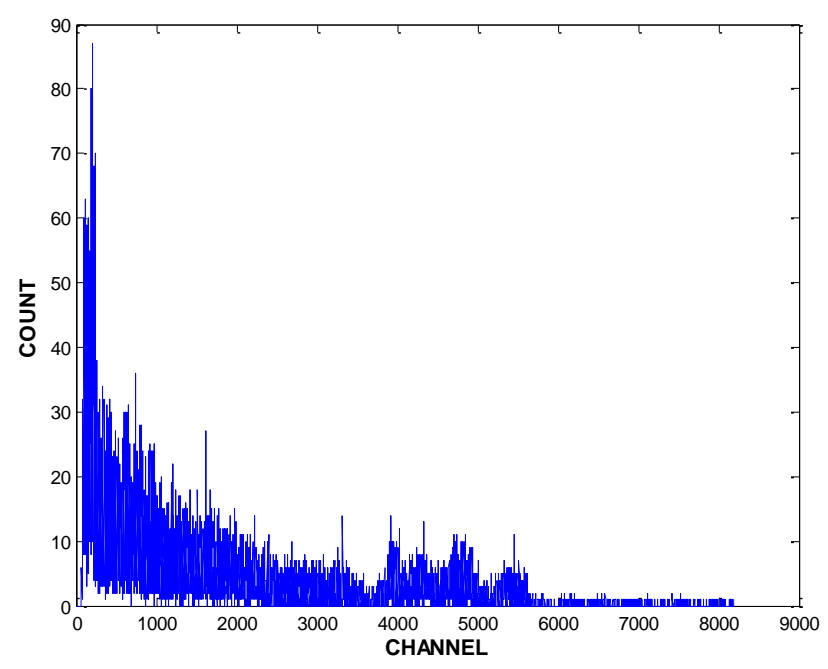

Figure 1: Gamma Ray Spectra

From this type of spectra, it is difficult to identify the peak and impossible task for very small peaks. To identify the peaks, the spectrum has to be smoothening. There are numerous strategies are accessible like Fast Fourier Transformation (FFT), Polynomial fitting, Gaussian, etc. Be that as it may, uses of these strategies are not generally viable, particularly if there should be an occurrence of escalated noised spectra as figure $(a)^{[1]}$. 
One of the challenges of computerized spectra analysis is to differentiate small peaks from random fluctuations in the multichannel data. Many smoothing algorithms available in order to attempt to eliminate random noise in the data that might be recognized as peaks by the peak search algorithm. There is a different purpose of gamma rays and mostly are used for experiment on the earth.

In the proposed approach for smoothing two methods are used which are B-spline and MBC. B-spline basis function can eliminate statistical fluctuation thoroughly with little data distortion and give curve which is smoothed. However smoothing decrease the variance of the raw counts at the cost of introducing some bias that de-emphasizes the peaks and valleys of spectrum. To achieve small RMSE over the entire spectrum, there is a need of MBC. MBC provides an effective compromise between peak fitting and the degree of off peak smoothing.

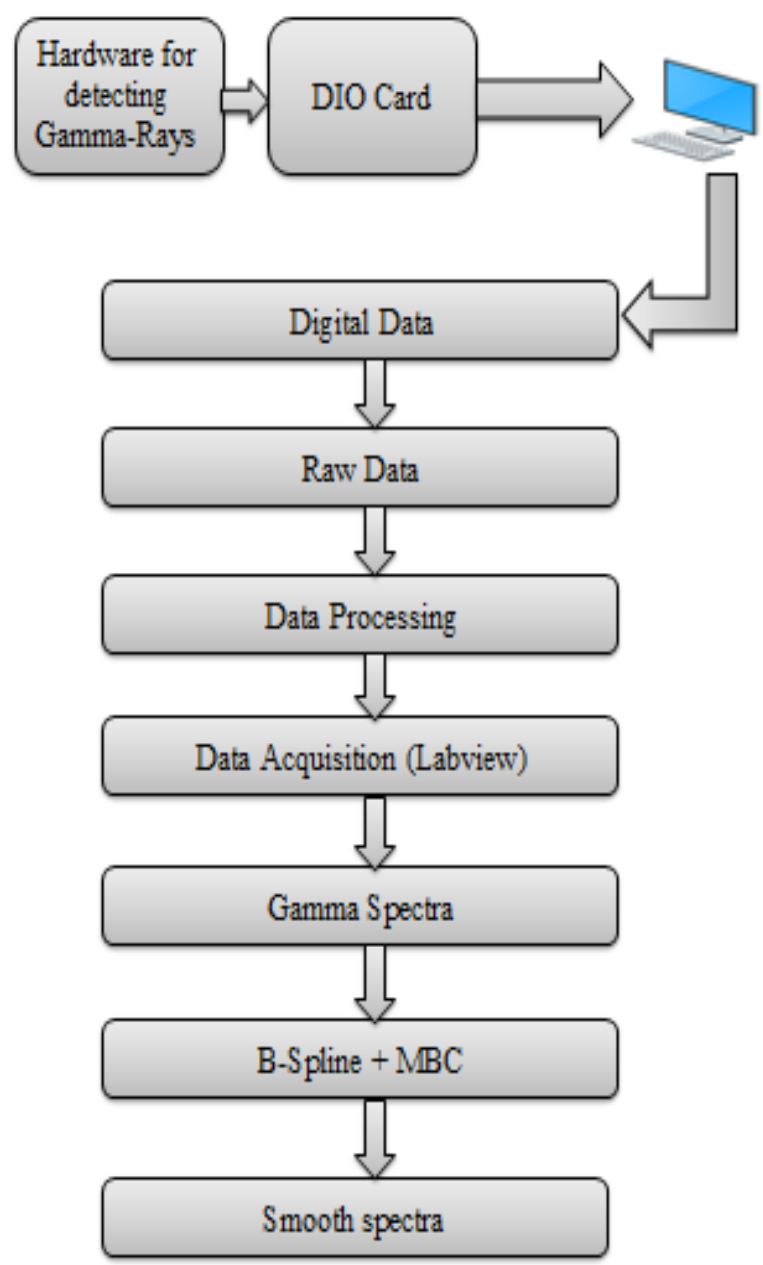

Figure 2: Proposed Approach

\section{RELATED WORK}

The work done by M.H.Zhu, L.G.Liu, Z. You, A.A.Xu ${ }^{[1]}$ utilized B-spline work for Least-Squares Fitting of Gammabeam Spectra. This technique depends on B-spline premise work with minimum squares fitting used to lessen the impact of measurable changes. This outcome demonstrates that this strategy is superior to conventional technique with a more total decrease of measurable variance. This slightest squares technique with B-spline premise capacity is acquainted with fit the gamma-beam spectra without re-preparing. Here better outcome will get while the estimation of math channel's full width at half maximum extreme is approximated to the normal FWHM of the crests in the spectra to be smoothed and it is elusive normal FWHM in the varied spectra. A slightest squares fitting strategy with B-splines premise work has been created for wiping out the factual change of gamma-beam spectra measured by indicator. Here the technique is straightforward and does not require any underlying information esteem. Diverse tests demonstrate that this strategy can expel measurable change totally with little information bending and the outcome best-fit bend is smooth which is critical out of sight disposal, crest seeking and recognizing the covering tops in the examination of gammabeam spectra.

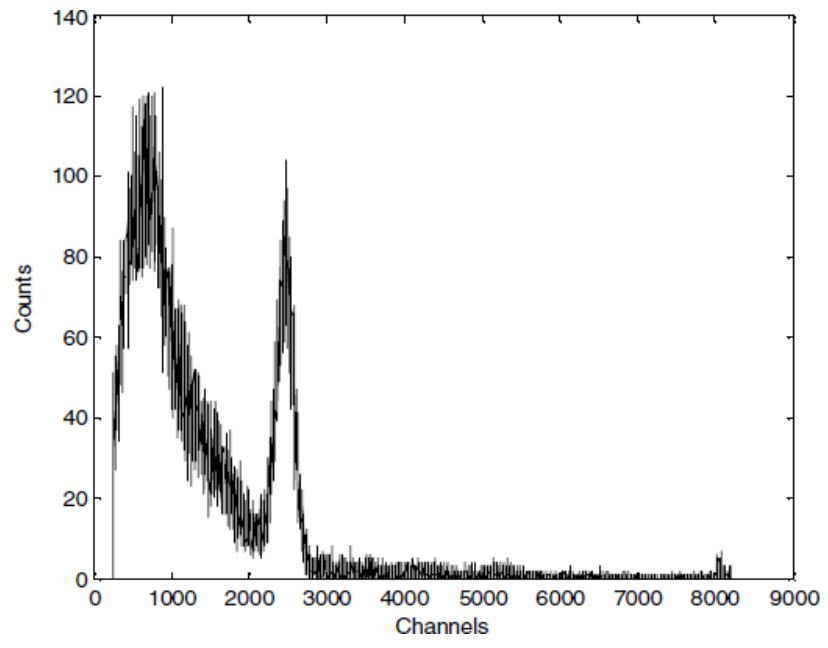

Figure 3: gamma-ray spectrum of 137Cs (NaI)

The B-spline premise capacity is characterized as a piecewise work which is non-zero just more than four neighboring interims between bunches.

$$
\emptyset(x)=\left\{\begin{array}{cc}
0 & |x| \geq 2 \\
\frac{1}{2}|x|^{3}-x^{2}+\frac{2}{3} & |x| \leq 1 \\
-\frac{1}{6}|x|^{3}+x^{2}-2|x|+\frac{4}{3} & 1<|x|<2
\end{array}\right.
$$

After applying this method, below spectrum is generated.

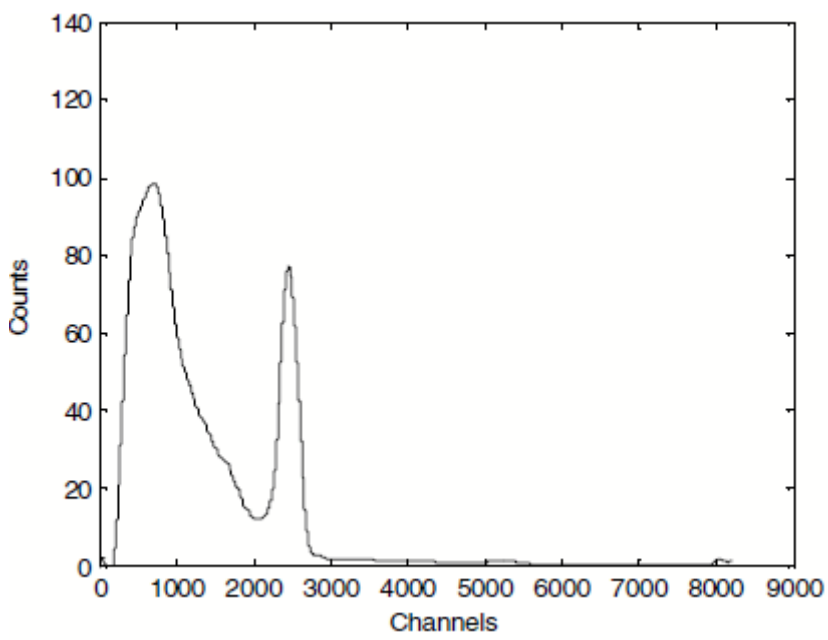

Figure 4: Smoothed data of ${ }^{137} \mathrm{Cs}$ 
The work done by Vincent Barnabé-Lortie, Colin Bellinger and Nathalie Japkowicz ${ }^{[4]}$ utilized machine learning idea for the smoothing of spectra. Here different method like Outlier detection, One-class Learning is used for smoothing Gammaray Data. There are many techniques for the outlier detection rather than framing it as classification problem like Statistical Testing Based methods, Depth-based methods, Distance based approaches and Density based approaches. In this paper they are including more strides which is preprocessing step smoothing with multiplicative predisposition revision going before the preparation of a classifier and testing of its execution. Here the gamma-beam spectra information are submitted to the preprocessing procedure before being part into preparing and testing sets or overlap for cross-approval.

Once smoothing spline has been fitted to a range, they apply the multiplicative predisposition remedy to re-accentuate the pinnacles and valleys. This is finished by taking the proportion of the underlying range and its smoothing splines, applying a smoother to that proportion, then reapplying the smoothed proportion multiplicatively to the smooth range.

\section{Algorithm: Smoothing preprocessing pseudocode}

$$
\begin{aligned}
& \text { firstPass } \leftarrow \text { cubicSplines (originalSpectrum) } \\
& \text { ratios } \leftarrow \text { originalSpectrum } \oslash \text { firstPass } \\
& \text { smoothRatios } \leftarrow \text { cubicSplines }(\text { ratios }) \\
& \text { secondPass } \leftarrow \text { smoothRatios } \odot \text { firstPass }
\end{aligned}
$$

The work done by T. Burr, N.Hengartner, E. Matzner-Lober, S. Myers and L. Rouviere ${ }^{[3]}$ utilized radio-isotopes ID (RIID) calculation. Systems for enhancing RIID by decreasing clamor in the information are being considered. Here one commotion decrease procedure is to cover the unearthly information up neighboring vitality receptacles. In any case, smoothing diminish, the change of the line checks at the cost of presenting some predisposition that de-stresses the pinnacles and valleys of the range. In this paper different smoothing techniques are used like Local Regression Smoother, Wavelet Smoother, Spline Smoother. This review shows advantage in smoothing spectra on the grounds that smoothing presents inclination in pinnacle and valley district. Here contend for a straightforward MBC smoother to alter an underlying smoother for inclination in the pinnacles and valleys. Thus, the main smoother does exceptionally well for expansive pinnacles or level locales, and the MBC connected to the principal smoother does basically the same on the wide pinnacles and pads areas, and much better in the more keen pinnacle districts. Quantitatively when connected to any sensible beginning smoother, MBC diminishes predisposition with immaterial or no expansion in difference, subsequently bringing about enhanced smoothing execution as measured by the root mean squared blunder.

The work done by Shiven Sharma, Colin Bellinger and Nathalie Japkowicz ${ }^{[2]}$ utilized Machine Learning Perspective. In this paper also used one class learning, The Autoassociator, The Autoassociator, Support Vector Machine, Mahalanobis Distance, Variance in Angle Spectrum. The fundamental rationale of this paper is to devise a robotized framework that could recognize and caution suitably on atypical gamma-beam phantom readings with little, if any intercession with respect to the human faculty included.

The work done by Thomas H. Prettyman, William C. Feldman, Kenneth R. Fuller, Steven A. Storms, Stephen A.
Soldner, Csaba Szeles, Frank P. Ameduri, David J. Lawrence, Michael C. Browne and Calvin E. Moss ${ }^{[5]}$ utilized GammaRay Spectrometer for Orbital Planetary Missions. New planetary science missions are being wanted to investigate Mercury, Mars, the space rock belt, and the external planets. Like Lunar Prospector, the greater part of these missions will utilize both neutron and gamma-beam spectrometers to outline plenitude. Be that as it may, because of mission limitations (particularly mass and warmth stacking), some of these missions will oblige instrumentation to be mounted on the deck of the shuttle, as opposed to on a blast. For instance, missions made arrangements for Mercury, including Bepi Columbo (ESA) and Messenger (NASA), will fly deckmounted spectrometers.

\section{CONCLUSION}

Data acquisition for gamma ray spectrometer is intended to serve as a tool to Scientists who need to describe the properties of the planet during the planetary mission. It consist the dataset of gamma rays and it has been used to study surface composition of the Moon, Mars and asteroids. With the help of smoothing of gamma rays spectra, it is easy to study information during planetary mission. For future work, the datasets of gamma rays acquired from DIO to study the surface composition of planets can be smoothening at initial stage itself. The dataset can be automatically smooth, reducing the complexity and time frame for processing raw dataset. This will give ready to use dataset to Scientists.

\section{ACKNOWLEDGMENT}

I would like to thanks to Mr. Dipak Kumar Panda (ScientistSE), Physical Research Laboratory (PRL), Ahmedabad for his precious time, help, valuable guidance, suggestions and sorting out the difficulties of my topic that helped me a lot, and continuous encouragement throughout the project and also for giving me such a nice opportunity to work in the great environment

\section{REFERENCES}

[1] M. H. Zhu, L. G. Liu, Z. You and A. A. Xu, "LeastSquares Fitting of Gamma-Ray Spectra with B-Spline Basis Functions," Image and Signal Processing, 2008. CISP '08. Congress on, Sanya, Hainan, 2008, pp. 691695.

[2] S. Sharma, C. Bellinger, N. Japkowicz, R. Berg and K. Ungar, "Anomaly detection in gamma ray spectra: A machine learning perspective," 2012 IEEE Symposium on Computational Intelligence for Security and Defence Applications, Ottawa, ON, 2012, pp. 1-8.

[3] T. Burr, N. Hengartner, E. Matzner-Lober, S. Myers and L. Rouviere, "Smoothing Low Resolution Gamma Spectra," in IEEE Transactions on Nuclear Science, vol. 57, no. 5, pp. 2831-2840, Oct. 2010.

[4] V. Barnabé-Lortie, C. Bellinger and N. Japkowicz, "Smoothing gamma ray spectra to improve outlier detection," the 2014 Seventh IEEE Symposium on Computational Intelligence for Security and Defense Applications (CISDA), Hanoi, 2014, pp. 1-8.

[5] T. H. Prettyman et al., "CdZnTe gamma-ray spectrometer for orbital planetary missions," in IEEE Transactions on Nuclear Science, vol. 49, no. 4, pp. 1881-1886, Aug 2002. 
[6] E. P. Binnall, "Instrumentation and Computer Based Data Acquisition for in-Situ Rock Property Measurements," in IEEE Transactions on Nuclear Science, vol. 27, no. 4, pp. 1291-1298, Aug. 1980.

[7] J. Anderson et al., "Data Acquisition and Trigger System of the Gamma Ray Energy Tracking In-Beam Nuclear Array (GRETINA)," in IEEE Transactions on Nuclear Science, vol. 56, no. 1, pp. 258-265, Feb. 2009.

[8] C. Granja, J. Jakubek, Y. Kopatch, S. Pospisil, S. Telezhnikov and Z. Vykydal, "Position-, spectral- and time-sensitive spectroscopy of fission fragments with TimePix pixel detectors," 2008 IEEE Nuclear Science Symposium Conference Record, Dresden, Germany, 2008, pp. 1659-1660.

[9] J. F. Amann, R. L. Boudrie, H. A. Thiessen, C. L. Morris and L. E. Smith, "Data Acquisition and Analysis on the
High Resolution Magnetic Spectrometers at LAMPF," in IEEE Transactions on Nuclear Science, vol. 26, no. 4, pp. 4389-4394, Aug. 1979.

[10] D. K. Panda, D. Banerjee, S. K. Goyal, A. R. Patel and A. D. Shukla, "Development of a cerium-doped lanthanum bromide gamma-ray spectrometer for planetary missions and feasibility studies for determination of elemental abundances of radioactive elements (Th, $\mathrm{K}$ and $\mathrm{U}), "$ in RESEARCH COMMUNICATION, Current Science, VOL. 110, NO. 11, 10 JUNE 2016

[11] W. C. Feldman, B. L. Barraclough, K. R. Fuller, D. J. Lawrence, S. Maurice, M. C. Miller, and T. H. Prettyman, "The Lunar Prospector gamma-ray and neutron spectrometers," Nucl. Instrum. Methods, vol. A422, no. 1-3, pp. 562-566, Feb. 1999. 